\title{
Characterisation of Liver Lesions on Triple Phase Computed Tomography and Studying the Social Demographics of the Liver Lesions
}

\author{
Anil Kumar Shukla ${ }^{1}$, Javaji Ravi Prasad ${ }^{2}$, Prashanth J ${ }^{3}$ \\ ${ }^{1}$ Professor, Department of Radiodiagnosis and Imaging, Rajarajeswari Medical College and Hospital, Kambipura, Mysore Road, Bengaluru- 560074, Karnataka, \\ India, ${ }^{2}$ Professor and HOD, Department of Radiodiagnosis and Imaging, Rajarajeswari Medical College and Hospital, Kambipura, Mysore Road, Bengaluru- \\ 560074, Karnataka, India, ${ }^{3}$ Junior Resident, Department of Radiodiagnosis and Imaging, Rajarajeswari Medical College and Hospital, Kambipura, Mysore Road, \\ Bengaluru- 560074, Karnataka, India.
}

\section{Abstract}

Background: To assess and characterize liver lesions using triple phase multi-detector computed tomography (MDCT) as a tool and to study the social demographics of the patients with liver lesions. Subjects and Methods: A hospital based retrospective analysis of 150 patients was done on patients with liver lesions who had undergone triple phase MDCT to characterize the liver lesions as was advised post ultrasonography or based on clinical suspicion, in a period of 3 years, from January 2015 to December 2017 in Rajarajeswari medical college and hospital and findings of triple phase MDCT were correlated with clinical/ histopathological or post-operative findings. The results were then analysed to arrive at conclusions. Results: Of the total of 150 patients with liver lesions. there were 104 benign and 46 malignant lesions. Hemangioma and metastasis were seen to be the most common lesions, with hemangioma accounting for 35 cases (24\%) and metastasis 28 cases $(18.6 \%)$. There were 17 cases (12\%) each of abscess and simple cysts, 21 (14\%) cases of hepatocellular carcinoma and 17 cases $(11 \%)$ of hydatid cysts. There were 8 cases (5\%) cases of focal fatty infiltration, 3 cases cases of hepatic laceration, $1 \%$ cases each of focal nodular hyperplasia, cholangiocarcinoma and hepatic contusion. Conclusion: Triphasic MDCT, owing to its strength of high speed mapping of the liver in the immediate arterial, portal venous and delayed phase assessing the enhancement pattern of lesions is an accurate and highly sensitive tool in diagnosing and characterising liver lesions.

Keywords: Liver, Triple Phase Computed Tomography.

Corresponding Author: Dr Javaji Ravi Prasad, Professor and HOD, Department of Radio-diagnosis and Imaging, Rajarajeswari medical college and Hospital, Kambipura, Mysore Road, Bengaluru- 560074, Karnataka, India.

Received: June 2019

Accepted: June 2019

\section{Introduction}

Detecting and characterizing focal liver lesions is one of the most difficult challenges in imaging today. Although histopathology is the gold standard, biopsy is always not possible as it is an invasive technique. All standard noninvasive imaging modalities are less sensitive than generally perceived, and characterization is imperfect. Though ultrasound has its strengths like its ability to characterize common benign lesions (eg: cysts and hemangiomas), safety, low cost, and its ability to guide biopsy, it has its own share of weaknesses like inability to image the entire liver in many patients and its inferiority to $\mathrm{CT}$ in sensitivity in detecting and diagnosing focal lesions. Hence, computed tomography (CT) is the imaging modality most often used to evaluate focal liver lesions, however, the complex blood supply of the liver necessitates the search for an optimal contrast-enhanced CT protocol for the detection and characterization of focal hepatic lesions. Consequently, the preferred liver CT technique should combine a high sensitivity for lesion detection with a good ability for lesion characterization, to differentiate lesions that do need further diagnostic tests or treatment for lesions that do not.

To meet these requirements, a triphasic multidetector CT (MDCT) technique was developed to image the entire liver in arterial, portal, and equilibrium phase. Triple phase MDCT is based on various enhancement patterns of lesions in different phases. On Non enhanced Computed Tomography (NECT) liver tumors usually are not visible. In the arterial phase hyper vascular tumors will enhance. In the portal venous phase hypo vascular tumors are detected. In the equilibrium phase these lesions will become hyperdense or hypodense to the normal liver.

This study of characterization of focal liver lesion will help to better understand complex liver pathologies and enhancement characteristics of different array of focal liver lesions.

\section{Aims and Objectives}

To study the role of triple phase MDCT scan in detection, diagnosis and differentiation of liver lesions.

- To study the enhancement patterns of various liver lesions on triple phase MDCT scan.

- To study the demographics of patients with liver lesions. 


\section{Subjects and Methods}

This is hospital based retrospective study of 150 cases. Study population included all patients with suspicion of hepatic masses on clinical, laboratory or ultrasonography findings. Cases of all age groups were included irrespective of sex. Exclusion criteria was patients with renal failure or those with history of allergic reactions to contrast, pregnant and claustrophobic patients.

CT scans were performed on Siemens Somatom Perspective 128 slice CT machine.

\section{Technique}

Arterial phase was taken at $35-40 \mathrm{sec}$ after contrast injection or 15-20 sec after bolus tracking. Lesions supplied by hepatic artery enhanced maximally in this phase

Hepatic or late portal phase was obtained at 70-80 sec after contrast injection or 50-60 sec after bolus tracking. Hepatic veins enhanced in this phase with maximal enhancement of hepatic parenchyma. Hypo-vascular lesions were best evaluated in this phase.

Delayed or equilibrium phase was obtained 2-10 minutes after the contrast injection. Those tumors became visible in this phase that either loosed their contrast slower than normal liver parenchyma or washed out rapidly.

Contrast agent used in the study was Iohexol (omnipaque) $350 \mathrm{mgI} / \mathrm{ml}$ i.v. Dose of intravenous contrast varied according to weight of the patient (1.2-1.5 cc per $\mathrm{kg}$ ) The injection rate was kept between $2.5-5 \mathrm{ml} / \mathrm{sec}$, rate was adjusted as such that complete contrast is administered in approximately $30 \mathrm{sec}$. (for $125 \mathrm{~mL}$ of contrast with $4 \mathrm{~mL} / \mathrm{sec}$ and for $150 \mathrm{~mL}$ with $5 \mathrm{~mL} / \mathrm{sec}$ ).

\section{Results}

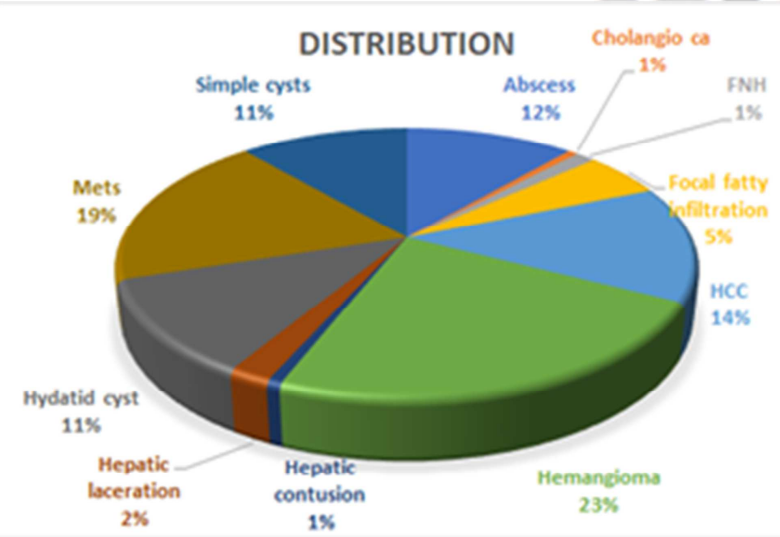

Figure 1: Pie chart showing the frequency of various liver lesions.

In our study, there were 104 benign lesions and 46 malignant lesions out of 150 patients. Hemangioma and metastasis were the most common lesions, with hemangioma accounting for 35 cases (24\%) and metastasis 28 cases $(18.6 \%)$. There were 17 cases $(12 \%)$ each of abscess and simple cysts and $21(14 \%)$ cases of hepatocellular carcinoma and 17 cases $(11 \%)$ of hydatid cysts. There were 8 cases $(5 \%)$ cases of focal fatty infiltration, 3 cases of hepatic laceration, $1 \%$ cases each of focal nodular hyperplasia, cholangiocarcinoma and hepatic contusion.

In our study, the study population comprised of cases with the presenting age ranging from 10 years to 85 years of age, with the maximum number of cases in the age group of 41 to 50 years $(30 \%)$. Further, $66 \%$ of cases were males and $34 \%$ females.

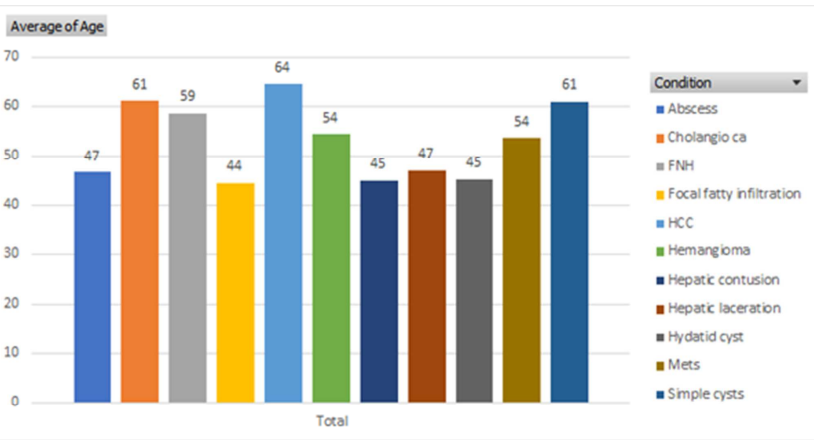

Figure 2: Histogram representing the average age of presentation of patients with various liver lesions.

In our study, it was observed that benign conditions generally had a younger average age at the time of presentation, such as abscess (46 years), focal fatty infiltration (44 years), hemangioma (55 years), hepatic contusions (45 years) whist malignant conditions such as cholangiocarcinoma (61 years), hepatocellular carcinoma (64 years) and metastasis (56 years) had an older average presentation ages.

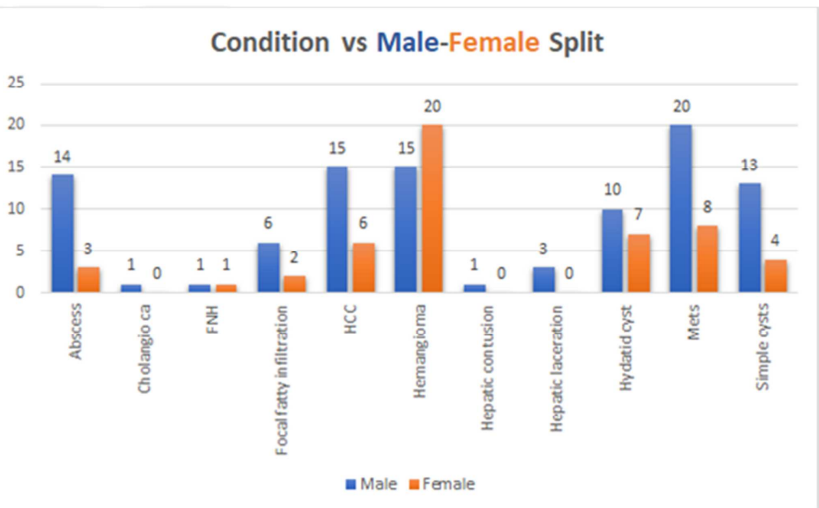

Figure 3: Histogram representing the male: female incidences of liver lesions in the study population

While analysing the male: female preponderance of liver diseases, it was noted that males were at a higher probability to have most of the liver lesions, benign and malignant lesions alike, such as abscesses (4.6:1), simple cysts $(3.25: 1)$ and hepatocellular carcinoma $(2.5: 1)$ etc. The only exception to this trend was in the case of hemangiomas, where there was a slight female preponderance (1:1.2). Incidentally, in our study, the traumatic lesions, i.e hepatic contusions and lacerations, were found only in males.

$\underline{\text { Representative Images }}$ 

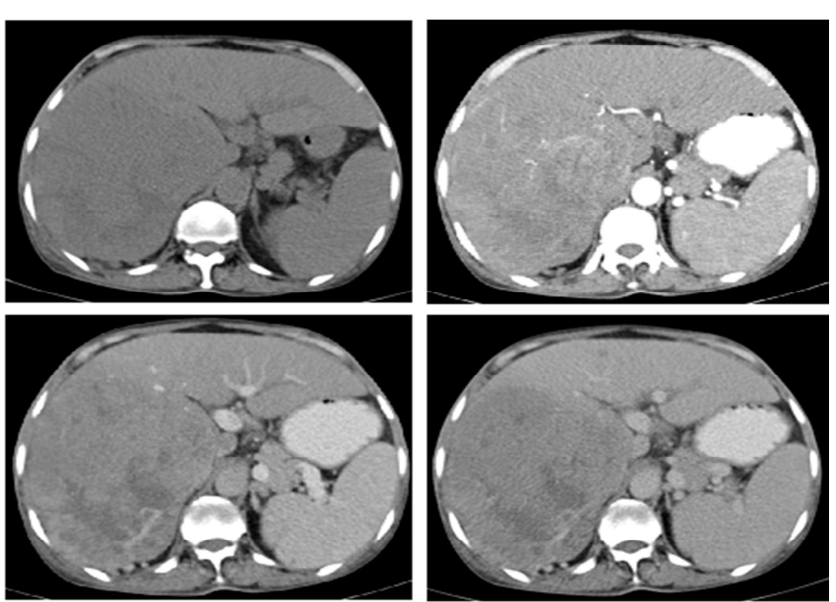

Figure 4: A case of hepatocellular carcinoma, (A)- plain CT showing irregular, predominantly hypodense lesion showing enhancement on arterial phase (B) and iso to hypo enhancement on portal venous (C) and delayed phase(D) (yellow arrow). Few non enhancing areas suggestive of necrosis seen within.
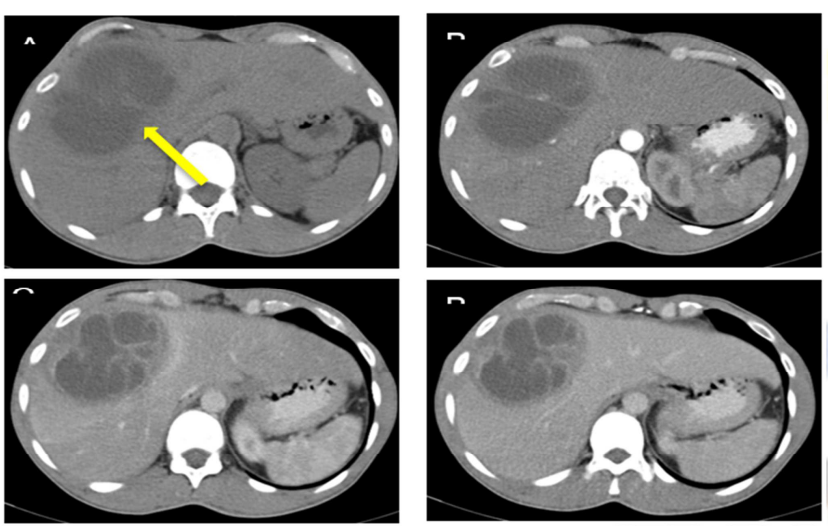

Figure 5: A case of liver abscess. A thick walled hypodense lesion with internal septations seen in the segments VII and VIII of liver on plain CT $(\Lambda)$ which shows peripheral capsular and septal enhancement on arterial (B), portal venous $(C)$ and delayed (D) axial sections.
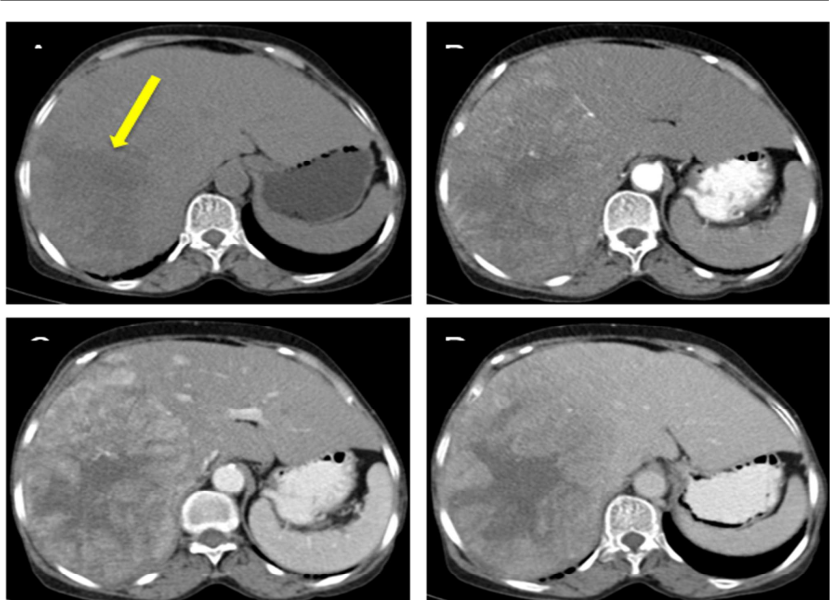

Figure 6: Another case of liver abscess (A)- An I'll defined hypodense lesion in the right lobe of liver showing heterogenous enhancement in arterial (B) and portal venous (C) and washout in the delayed phase (D). There are multiple non enhancing areas within, suggestive of necrotic areas.
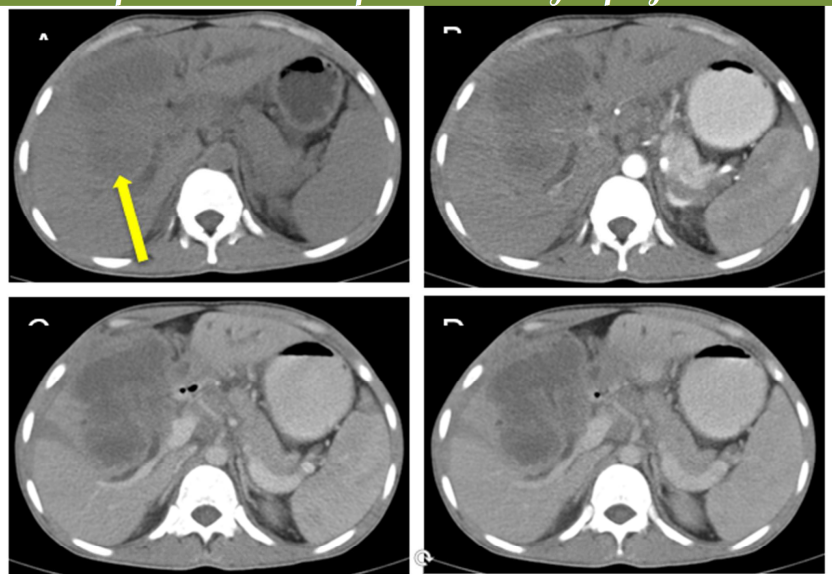

Figure 7: A case of Cholangiocarcinoma -. A large ill-defined, hypodense lesion on plain scan (A) is noted in the segments $I$, IVB and $V$ showing inhomogeneous enhancement on arterial (A), portal venous (C) and delayed phase (D) images. Also seen are dilated right main hepatic duct and intrahepatic biliary duct dilatation.
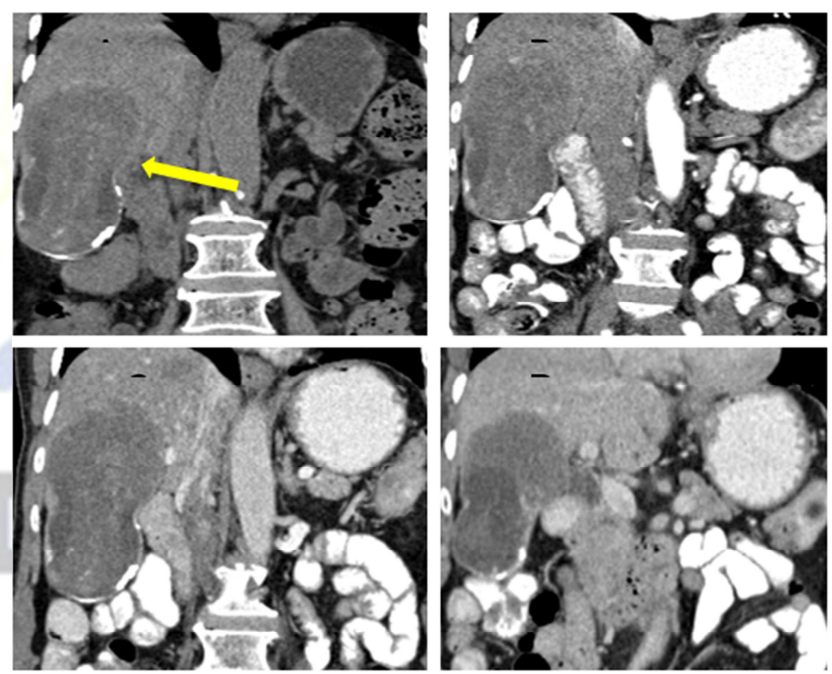

Figure 8: A case of hydatid cyst- large well defined heterodense lesion noted in segment VI and VII of right lobe of liver with an exophytic component showing both cystic (daughter cysts) and solid component with peripheral and internal calcifications on plain scan (A). Solid component shows minimal enhancement post contrast $(B, C$ and $D)$.

\section{Discussion}

\section{Hemangiomas}

In our study, hemangioma was the most common liver lesion with 35 cases, of which 20 cases were females and 15 males, the only condition to have a female predominance. This is in agreement with Aytekin et al, who had described that hemangioma are more common in females. ${ }^{[1,2]}$ All the cases were hypodense on plain scan. On post contrast scans, all the lesions (except one) showed enhancement in arterial phase followed by gradual filling toward the centre and prolonged enhancement on the equilibrium phase. All the hemangiomas were solitary and a majority of lesions (32) were less than $10 \mathrm{~cm}$ in size, only 3 lesions (8.5\%) were 
more than $10 \mathrm{~cm}$. Mayo Foundation for Medical Education and Research, 2011 has described that most of the hepatic hemangioma are small, single and do not produce symptoms. Triple phase CT scan correctly diagnosed all the cases $(100 \%)$ of hemangiomas

Metastasis: Metastasis was the second most common liver lesion in our study with 28 cases $(18.6 \%)$. There were 20 males and 8 females with metastasis. Incidentally, the 2 youngest patients in the study, aged 11 and 13. were cases of liver metastasis. The primary tumour in the former was Wilm's tumour of the left kidney and in the latter, dysgerminoma in the retroperitoneum. Hypodense lesion on non-contrast $\mathrm{CT}$ and less enhancement than surrounding liver on postcontrast studies is the most common appearance of hepatic metastases. This was in accordance with a study done by Soyer P64 Foley et al, had described similar findings. 3 Most of the cases (26 cases) had lesions showing well defined margins (93\%). 2 cases demonstrated enhancement in the late arterial phase with peripheral continuous enhancement suggestive of hyper vascular metastasis. Absence of contrast washout in delayed phase and nodular peripheral centripetal enhancement are useful to differentiate hemangiomas from hyper vascular liver metastases. This is in accordance with a study done by D F Leslie61 and Kim T56.

Hepatocellular carcinoma: There was a total of 21 cases of hepatocellular carcinoma, constituting $14 \%$ of total number of cases. All patients had mass of size more than 20 mm. 19 cases had lesions with well-defined margins (90.4\%) and 2 (9.6\%) had ill-defined margins. All lesions showed the typical pattern of early enhancement in arterial phase with rapid washout in Porto venous phase. All lesions were hypodense in delayed phase. In 13 (62\%) patients portal vein thrombosis was seen. Saini et al, has described that the tumor thrombus is another one of the characteristic features of HCC4. In two patients, portal cavernoma formation was noted due to tumor in portal vein. Capsular enhancement on delayed phase images was noted in 15 patients $(71 \%)$. Similar findings were described by Lee et al. ${ }^{[5]}$

Abscess: There were 14 males and 3 females of the total 17 cases of hepatic abscess. Most of the abscesses were well defined and 10 cases $(58.8 \%)$ showed presence of multiple septa within the lesion. In 2 cases, there were multiple small cystic masses clustered together. On non-enhanced MDCT, the abscesses appeared hypodense and showed peripheral rim, capsule and septal enhancement on triple phase MDCT. 3 patients $(17.6 \%)$ showed air foci within the abscess cavity. Three cases were accompanied by transient hepatic attenuation difference (THAD). In 7 patients, there was accompanying pleural effusion and further right lower lobe atelectasis in 2 patients.

Simple cyst/cysts: On triple phase MDCT scan, they appeared as non-enhancing sharply defined hypodense lesions showing water density (HU: -10 to +10 ). In 3 cases $(17 \%)$ of the total 17 , presence of multiple simple cysts was noted.

Hepatic trauma: There were 3 patients with laceration and 1 with contusion, incidentally all the patients with trauma being males All 3 patients with laceration had associated subcapsular hematoma, with 2 of them having right sided multiple rib fractures. All patients had associated hemoperitoneum.

Hydatid cyst: On MDCT hydatid cyst was noted as well defined cystic lesion. 2 patients showed daughter cysts within the main cyst.

Focal fatty infiltration: All 8 cases showed well defined lesions which showed decreased attenuation compared to that of spleen on non-enhanced MDCT, followed by a difference of $>35 \mathrm{HU}$ on venous phase compared to that of spleen attenuation.

Cholangiocarcinoma: There was only 1 case of cholangiocarcinoma (61-year-old male). The patient presented with jaundice and hyperbilirubinemia. Bloom et al, has described similar features in their study.6 Ill-defined iso-hypodense lesion was seen in the right lobe of liver showing predominant enhancement in portal phase. Patchy hypodense areas with central necrosis seen within the lesion were seen. Similar findings were described by Nisha et al. ${ }^{[7]}$ Focal Nodular hyperplasia (FNH): There were 2 cases (1 male and 1 female) of FNH in our study. On non-enhanced MDCT, the lesion appeared well defined, lobulated and hypodense with more hypodense central scar. On triple phase MDCT, the lesion showed homogenous enhancement in arterial phase with non-enhancing central scar. On portal venous phase images, the lesion showed homogenous enhancement with non-enhancing central scar. On delayed phase images enhancement of scar was noted.

\section{Conclusion}

Triple phase MDCT is very effective in characterisation of focal hepatic lesions by accurately assessing size, location, enhancement patterns, necrosis, calcification and associated lymphadenopathy. It is less time consuming, cost-effective and highly sensitive. It can differentiate between benign and malignant lesions. Benign conditions generally have a younger average age at the time of presentation, whist malignant conditions have older average presentation ages. The incidence of liver lesions is higher in males than in females, the only exception to this being hemangiomas, where there is a female predominance.

\section{References}

1. Ahirwar CP, Patil A, Soni N. Role of triple phase computed tomography findings for evaluation of hepatic lesions. Int J Res Med Sci 2016:4:3576-83

2. Oto A, Kulkarni K, Nishikawa R, Baron RL. Contrast enhancement of hepatic hemangiomas on multiphase MDCT: Can We Diagnose hepatic hemangiomas by comparing enhancement with blood pool? AJR. 2010;195:381-6.

3. Foley D, Kerimoglu U. Abdominal MDCT: Liver, Pancreas, and Biliary Tract Seminars in Ultrasound, CT, and MRI. 2004;25:122-44.

4. Gazelle SG, Saini S, Mueller P. Hepatobiliary and pancreatic Radiology imaging and intervention. Thieme. 1998.

5. Lee KHY, O'Malley ME, Haider MA, Hanbidge A. Triple phase MDCT of hepatocellular carcinoma. AJR. 2004;182:643-49.

6. Bloom CM, Langer B, Wilson SR. Role of US in the detection, characterization, and staging of cholangiocarcinoma. RadioGraphics 1999;19:1199-218. 


\section{Shukla et al; Characterisation of Liver Lesions an Triple Shase Camputed Tamagraphy}

7. Sainani NI, Onofrio A, Catalano, Nagaraj Setty, Holalkere, Zhu AX, Hahn PF, Sahani DV. Cholangiocarcinoma: Current and Novel Imaging Techniques. RadioGraphics. 2008;28:1263-87.

8. David Sutton, Textbook of radiology and imaging, 7 th edition, Vol. 1.

9. Grainger, R.G. Allison D.J. Diagnostic radiology, 5th edition.

10. Hind S. Alsaif et al. CT Appearance of Pyogenic Liver Abscesses Caused by Klebsiella pneumonia; July 2011, Radiology, 260, 129-138.

11. Hsien-Ping Lin, Wei-Chi Ho, Cheng-Chi Lee, Kwok-Ting Lin, and Yu-Chiang Lin; Infected Simple Hepatic Cysts - 3 Cases Report; J Intern Med Taiwan, 2009, 20, 373-377

12. Leon Schiff, Eugene Schiff, Diseases of the liver, 6th edition, Lippincott.

13. Mortelé KJ, Ros PR.; Cystic focal liver lesions in the adult: differential CT and MR imaging features. Radio Graphics,2001; 21(4): 895-910.

14. Zakim, Boyer, Reviewed by D H Adams 2003. Hepatology: a Textbook of Liver Disease, 4th edition, Saunders. 48078 Dr. Divya Bagoria et al. Role of triple phase CT in evaluation of focal liver lesions

15. Anatomy and physiology of the liver - Canadian Cancer Society. Cancer.ca. Retrieved 2015-06-26.

16. Abdel-Misih SR, Bloomston M. Liver Anatomy. Surgical Clinics of North America. 2010;90(4):643-53

17. Sinnatamby CS. Last's Anatomy: Regional and Applied. 10th Ed. Edinburgh: Churchill Livingstone. 1999.

18. Foley WD, Mallisee TA, Hohenwalter MD, Wilson CR, Quiroz FA, Taylor AJ. Multiphase hepatic CT with a multirow detector CT scanner. AJR Am J Roentgenol. 2000;175:679-685.

19. Oliver JH, Baron RL, Federle MP, Rockette HE Jr. Detecting hepatocellular carcinoma: value of unenhanced or arterial phase CT imaging or both used in conjunction with conventional portal venous phase contrast-enhanced CT imaging. AJR. 1996;167:71-7.

20. Atasoy C, Akyar S. Multidetector CT: contributions in liver imaging. Eur J Radiol. 2004;52(1):2-17.
21. Boll DT, Merkle EM. Liver: Imaging Techniques, and Diffuse Diseases. In: Haaga JR, Dogra VS, Forsting M, Gilkeson RC, Ha KH, Sundaram M editors. CT \&MRI of whole body. 5th ed. Mosby: Inc. 2009 .

22. Bartolotta TV, Midiri M, Galia M. Characterization of benign hepatic tumors arising in fatty liver with SonoVue and pulse inversion US. Abdom-Imaging. 2007;32(1):84-91.

23. Ichikawa T, Federle MP, Grazioli L, Nalesnik M. Hepatocellular adenoma: multiphasic CT and histopathologic findings in 25 patients. Radiology. 2000;214:861-8.

24. Blachar A, Federle MP, Ferris JV. Radiologists' performance in the diagnosis of liver tumors with central scars by using specific CT criteria. Radiology. 2002;223(2):532-39.

25. Kassarjian A, Zurakowski D, Dubois J, Paltiel HJ, Fishman SJ, Burrows PE. Infantile hepatic hemangiomas: clinical and imaging findings and their correlation with therapy. AJR Am J Roentgenol. 2004;182(3):785-95

26. Keslar PJ, Buck JL, Selby DM. Infantile hemangioendothelioma of the liver revisited. RadioGraphics. 1993;13(3):657-70.

27. Levy AD, Murakata LA, Abbott RM, Rohrmann CA Jr. From the archives of the AFIP. Benign tumors and tumorlike lesions of the gallbladder and extrahepatic bile ducts: radiologic-pathologic correlation. Armed Forces Institute of Pathology. Radiographics. 2002;22(2):387-413.

28. Palacios E, Shannon M, Solomon C, Guzman M. Biliary cystadenoma: ultrasound, CT, and MRI. Gastrointest Radiol. 1990;15:313-16.

29. Buetow PC, Midkiff RB. Primary malignant neoplasms in the adult. MagnReson Imaging Clin N Am. 1997;5:289-318.

30. Chung EM, Cube R, Lewis RB, Conran RM. Pediatric liver masses: radiologic-pathologic correlation part 1- benign tumors RadioGraphics. 2010;30:801-26.

Copyright: ( $\mathrm{C}$ the author(s), publisher. Asian Journal of Medical Radiological Research is an Official Publication of "Society for Health Care \& Research Development". It is an open-access article distributed under the terms of the Creative Commons Attribution Non-Commercial License, which permits unrestricted non-commercial use, distribution, and reproduction in any medium, provided the original work is properly cited.

How to cite this article: Shukla AK, Prasad JR, Prashanth J. Characterisation of Liver Lesions on Triple Phase Computed Tomography and Studying the Social Demographics of the Liver Lesions. Asian J. Med. Radiol. Res. 2019;7(1):68-72.

DOI: dx.doi.org/10.21276/ajmrr.2019.7.1.15

Source of Support: Nil, Conflict of Interest: None declared 